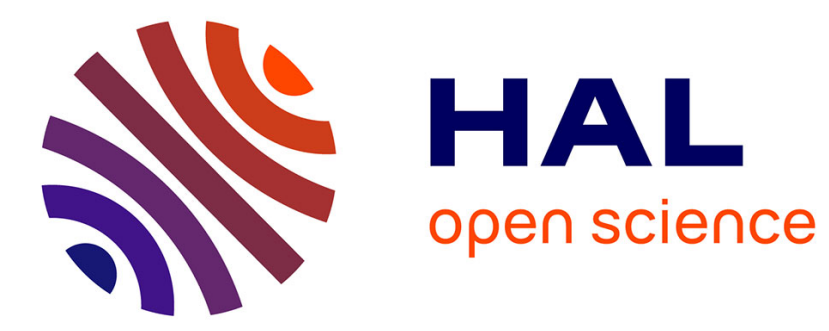

\title{
Adaptive attenuation of unknown and time-varying narrow band and broadband disturbances
}

Ioan Doré Landau, Tudor-Bogdan Airimitoaie, Abraham Castellanos Silva

\section{To cite this version:}

Ioan Doré Landau, Tudor-Bogdan Airimitoaie, Abraham Castellanos Silva. Adaptive attenuation of unknown and time-varying narrow band and broadband disturbances. International Journal of Adaptive Control and Signal Processing, 2015, 29 (11), pp.1367-1390. 10.1002/acs.2547 . hal-01168620

\section{HAL Id: hal-01168620 \\ https://hal.science/hal-01168620}

Submitted on 6 Oct 2020

HAL is a multi-disciplinary open access archive for the deposit and dissemination of scientific research documents, whether they are published or not. The documents may come from teaching and research institutions in France or abroad, or from public or private research centers.
L'archive ouverte pluridisciplinaire HAL, est destinée au dépôt et à la diffusion de documents scientifiques de niveau recherche, publiés ou non, émanant des établissements d'enseignement et de recherche français ou étrangers, des laboratoires publics ou privés. 


\title{
Adaptive Attenuation of Unknown and Time Varying Narrow Band and Broad Band Disturbances ${ }^{\dagger}$
}

\author{
Ioan Doré Landau ${ }^{1 *}$, Tudor-Bogdan Airimitoaie ${ }^{2}$, Abraham Castellanos Silva $^{1}$ \\ ${ }^{1}$ Control system department of GIPSA-LAB, 38402 St. Martin d'Héres, France \\ ${ }^{2}$ University of Bordeaux, IMS laboratory - UMR 5218 CNRS, Talence, 33405 France
}

\begin{abstract}
SUMMARY
In many classes of applications like active vibration control and active noise control, the disturbances can be characterized by their frequency content and their location in a specific region in the frequency domain. The disturbances can be of narrow band type (simple or multiple) or of broad band type. A model can be associated to these disturbances. The knowledge of this model allows to design an appropriate control system in order to attenuate (or to reject) their effect upon the system to be controlled. The attenuation of disturbances by feedback is limited by the Bode Integral and the "water bed" effect upon the output sensitivity function. In such situations, the feedback approach has to be complemented by a "feedforward disturbance compensation" requiring an additional transducer for getting information upon the disturbance. Unfortunately in most of the situations the disturbances are unknown and time-varying and therefore an adaptive approach should be considered. The generic term for adaptive attenuation of unknown and timevarying disturbances is "adaptive regulation" (known plant model, unknown and time-varying disturbance model).

The paper will review a number of recent developments for adaptive feedback compensation of multiple unknown and time-varying narrow band disturbances and for adaptive feedforward compensation of broad band disturbances in the presence of the inherent internal positive feedback caused by the coupling between the compensator system and the measurement of the image of the disturbance. Some experimental results obtained on a relevant active vibration control system will illustrate the performance of the various algorithms presented. Some open research problems will be mentioned in the conclusion. Copyright (C) 2015 John Wiley \& Sons, Ltd.
\end{abstract}

Received ...

KEY WORDS: Adaptive Regulation, Adaptive Feedforward Compensation, Active Vibration Control, Inertial Actuators, Youla-Kučera Parametrization, Internal Model Principle

\section{INTRODUCTION}

\subsection{The problem}

In many classes of applications like active vibration control (active suspension, control of disk drives) and active noise control, the disturbances acting upon a system and which have to be compensated (rejected, attenuated) can be characterized by their frequencies content and their

\footnotetext{
*Correspondence to: ioan-dore.landau@gipsa-lab.grenoble-inp.fr

${ }^{\dagger}$ A preliminary version of this paper has been presented as an invited plenary paper at the IFAC International Workshop on Adaptation and Learning in Control and Signal Processing in Caen, France, July 3-5, 2013.

¥This is the peer reviewed version of the following article: Ioan Doré Landau, Tudor-Bogdan Airimitoaie, and Abraham Castellanos Silva, Adaptive attenuation of unknown and time-varying narrow band and broadband disturbances, International Journal of Adaptive Control and Signal Processing, 29(11): 1367-1390, 2015, which has been published in final form at https://doi.org/10.1002/acs.2547. This article may be used for noncommercial purposes in accordance with Wiley Terms and Conditions for Use of Self-Archived Versions.
} 


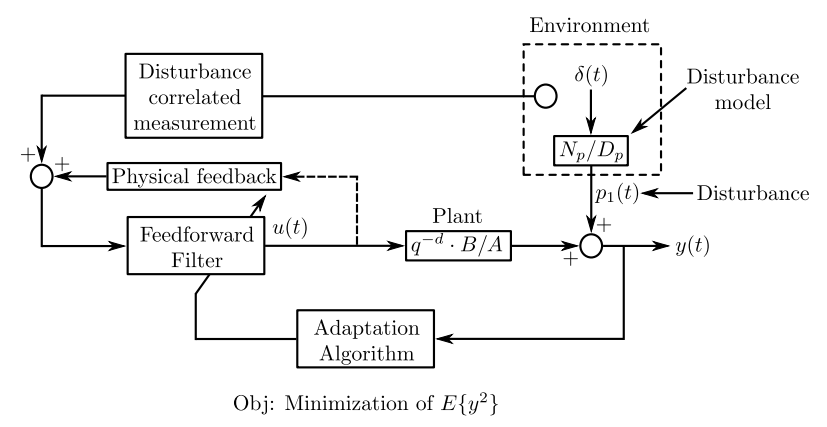

Figure 1. Adaptive feedforward compensation of unknown disturbances.

location in a specific region in the frequency domain. The disturbances can be of narrow band type (simple or multiple) or of broad band type. Of course a combination of both is possible and what we call broad band may be in certain cases finite band disturbances over a small region in the frequency domain. However the distinction between these two types of disturbances is convenient in order to examine the techniques used for their compensation.

Fundamentally in active control a compensator system is introduced which will generate a "secondary" source. This compensator (acting through "secondary path") conveniently driven will interfere destructively with the disturbance coming from the "original " primary source (in general non accessible) through which is called the "primary path". A common framework is the assumption that the disturbance is the result of a white noise or a Dirac impulse passed through the model of the disturbance. The knowledge of this model together with the knowledge of the model of the secondary path (compensator) allows the design of an appropriate control strategy.

However in practice in most of the cases the characteristics of these disturbances are unknown or time-varying. While in some particular cases a robust design can be considered [1,2], in most of the situations, as a consequence of the high level of attenuation requested, an adaptive approach is necessary for obtaining a good tuning with respect to the disturbance characteristics.

When considering the model of a disturbance, one has to address two issues: 1) its structure (complexity, order of the parametric model) and 2) the values of the parameters of the model. In general, one can assess from data the structure for such model of disturbance (using spectral analysis or order estimation techniques) and assume that the structure does not change. Therefore adaptation will have to deal with the change in the parameters of the model of the disturbance.

\subsection{The basic techniques}

Historically, it seems that the first approach to be used systematically for disturbance compensation was the adaptive feedforward approach [3]. Probably one of the first references is [4]. Fig. 1 illustrates the adaptive rejection of unknown disturbances by feedforward compensation. A "well located" transducer can provide a measurement, highly correlated with the unknown disturbance (a good image of the disturbance). This information is applied to the control input of the plant through an adaptive filter whose parameters are adapted such that the effect of the disturbance upon the output is minimized.

Adaptive feedforward vibration (or noise) compensation is currently used in ANC and AVC when an image of the disturbance is available [5, 6, 7, 8]. However, at the end of the nineties it was pointed out that in most of these systems there is a physical "positive" feedback coupling between the compensator system and the measurement of the image of the disturbance (vibration or noise) $[8,9,6,7,10]$. Nevertheless it is possible to achieve attenuation (rejection) of narrow band disturbances using only a feedback approach (no need for a correlated measurement with the disturbance). But since the model of the disturbance is unknown and/or time-varying an adaptive feedback approach has to be used.

So at this point one can say that we have two types of disturbances

- Single or multiple narrow band disturbances: 
- Broad (finite) band disturbances.

and two approaches for doing disturbance attenuation:

- Adaptive feedforward compensation requiring an additional transducer for getting a correlated measurement with the disturbance.

- Adaptive feedback approach (which requires only a measurement of the residual force, acceleration, noise).

Lets assume for the moment that the disturbances are constant and that the models of the disturbances have been identified. The question is: why use "feedforward" compensation which requires an additional transducer some times difficult to place and which may be subject to an inherent physical positive feedback leading to stability problems? The answer comes from the Bode Integral of the output sensitivity function (the transfer function between the disturbance and the output of the system). Since the objective is to strongly attenuate (even reject totally asymptotically) the disturbance, this may require significative holes in the magnitude of the sensitivity function which in turn (even with a very careful design) may lead to unacceptable "water bed" effect both in terms of performance (one amplifies at certain frequencies where some disturbance can still be present) as well as in terms of robustness (the modulus margin may become unacceptable ${ }^{1}$ ). Basically narrow band disturbances can be rejected by feedback up to a certain number (at least 3 see [12]). Finite band disturbances enough "narrow" can also be handled by feedback only. However broad band disturbance attenuation will require to use adaptive feedforward compensation despite the difficulties indicated earlier. In fact the good approach is to do as much as possible by feedback and add on top the feedforward compensation.

\subsection{A conceptual feedback framework}

Fig. 2 represents an active noise and vibration control (ANVC) system using both feedforward and feedback compensators. The system has two inputs and two outputs. The first input is the disturbance $w(t)$ which is generated by the unknown disturbance source $s(t)$ passed through a filter with unknown characteristics. The second input is the control signal, $u(t)$. The first output is the measurement of the residual acceleration (force, noise) $e(t)$ (also called the performance variable) and the second output is a signal correlated with the unknown disturbance, $y_{1}(t)$ in Fig. 2. This correlation is a result of the physical characteristics of the system. As shown in Fig. 2, the path that transmits the filtered disturbance, $w(t)$, to the residual acceleration is called the primary path. The control signal, on the other hand, is transmitted to the residual acceleration through the secondary path. The residual acceleration (performance variable) is formed by addition between the output of the primary path, denoted $x(t)$, and the output of the secondary path, denoted $z(t)$. ANVC systems present in general also positive coupling path (also called reverse path) between the control signal $u(t)$ and the measured $y_{1}(t)$, which is shown in Fig. 2. This results in an internal positive feedback which can destabilize the ANVC system if not taken into account. The objective is that of minimizing the performance variable, $e(t)$, by computing an appropriate control, $u(t)$, based on the measurements $e(t)$ and $y_{1}(t)$. One can see that, in the control system architecture presented in Fig. 2, the control signal $u(t)$ is obtained by the subtraction between the feedforward control, $u_{1}(t)$, and the feedback control, $u_{2}(t)$. The measurements obtained from the system can be put into a vector form as $y(t)=\left[y_{1}(t), y_{2}(t)\right]^{T}=\left[y_{1}(t), e(t)\right]^{T}$. As a consequence, the controller also has a vector representation $\kappa=[N,-K]^{T}$, where $N$ and $K$ denote respectively the feedforward and the feedback compensators ${ }^{2}$. With these notations, the equation relating the measurements to the control signal is given by

$$
u(t)=u_{1}(t)-u_{2}(t)=N \cdot y_{1}(t)-K \cdot y_{2}(t)=\kappa^{T} \cdot y(t) .
$$

\footnotetext{
${ }^{1}$ The modulus margin is the minimum distance between the open loop transfer function hodograph and the Nyquist point [11].

${ }^{2}$ Both feedforward compensator and feedback compensator may have a more complex structure using for example YoulaKučera parametrization. Feedback controller may include explicitly a disturbance observer.
} 


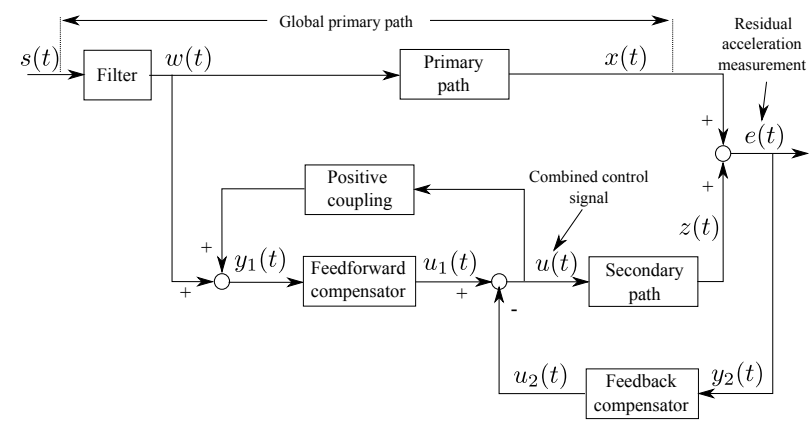

Figure 2. Block diagram representation of the combined feedforward-feedback control problem.

The feedforward controller denomination attributed to $N$ is motivated by the fact that $y_{1}(t)$, also called correlated image of the disturbance, is measured upstream of the performance variable. This assumes also that it is physically possible to obtain such a measurement. The situations where this is not possible constitute feedback control problems, while the others are more generally addressed in the literature as hybrid control. A standard feedback representation in the form of a 2 inputs - 2

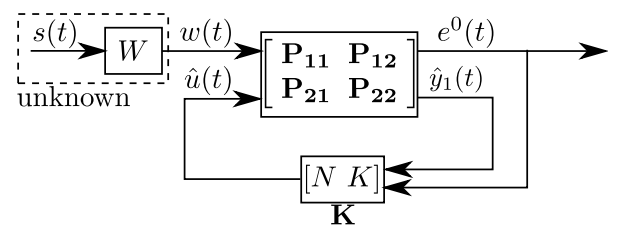

Figure 3. Generalized ANVC system representation.

outputs system can also be considered as shown in Fig. 3. This representation is very well known in robust and optimal control (see also [13]). The equations associated with the feedback system representation are

$$
\left[\begin{array}{l}
e(t) \\
y(t)
\end{array}\right]=\left[\begin{array}{ll}
P_{11} & P_{12} \\
P_{21} & P_{22}
\end{array}\right]\left[\begin{array}{l}
w(t) \\
u(t)
\end{array}\right]=\left[\begin{array}{c|c}
D & G \\
\hline 1 & M \\
D & G
\end{array}\right]\left[\begin{array}{l}
w(t) \\
u(t)
\end{array}\right],
$$

where $\mathrm{D}, \mathrm{G}$ and $\mathrm{M}$ correspond to the models of the primary, secondary and reverse paths. The control is given by (1).

\subsection{The adaptive regulation paradigm}

Consider the case of attenuation (rejection) of disturbances by feedback only. Since the parameters of the disturbance model are unknown and/or time-varying in order to get satisfactory performance an adaptive feedback approach has to be considered. The classical adaptive control paradigm deals essentially with the construction of a control law when the parameters of the plant dynamic model are unknown and time-varying ([10]). However, in the present context, the plant dynamic model is almost invariant and it can be identified. The objective then is the rejection of disturbances characterized by unknown and time-varying disturbance models. It seems reasonable to call this paradigm adaptive regulation. It is also assumed that the possible small variations or uncertainties of the plant model can be handled by a robust control design. The problem of adaptive regulation as defined above has been previously addressed in a number of papers $([14,15,16,17,18,19,20$, $21,22,23,24]$ ) among others. [12] presents a survey of the various techniques (up to 2010) used in adaptive regulation as well as a review of a number of applications.

An international benchmark has been organized on the attenuation of multiple and unknown time varying narrow band disturbances. The test bed was an active vibration control system. The results are summarized in [25]. However the adaptive regulation covers also the case of feedforward since 
on one hand adaptation has to deal with the change in the characteristics of the disturbances and on the other hand it is still a feedback structure as shown before.

The paper is organized as follows. Section 2 will present an active vibration control system used to test the various algorithms for disturbance attenuation which will be presented in the paper. Section 3 will review some basic algorithms for the attenuation of multiple unknown an time varying narrow band disturbances. Section 4 will present an experimental comparison of the performance obtained with the direct an indirect adaptive regulation approach. Section 5 will discuss the adaptive attenuation of broad band disturbances by feedforward and feedback. Section 6 gives a summary of experimental results for attenuation of broad band disturbances using adaptive feedforward with or without additional feedback control.

\section{AN ACTIVE VIBRATION CONTROL SYSTEM USING AN INERTIAL ACTUATOR}

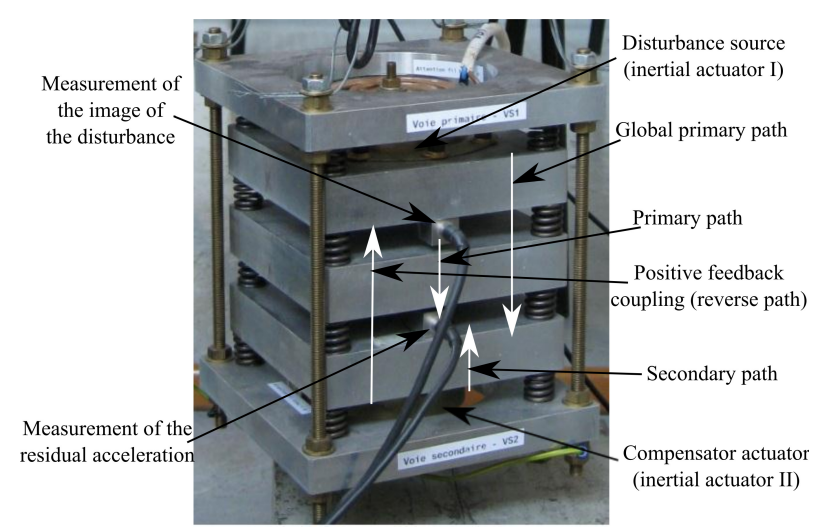

Figure 4. An AVC system using a feedforward compensation - photo.

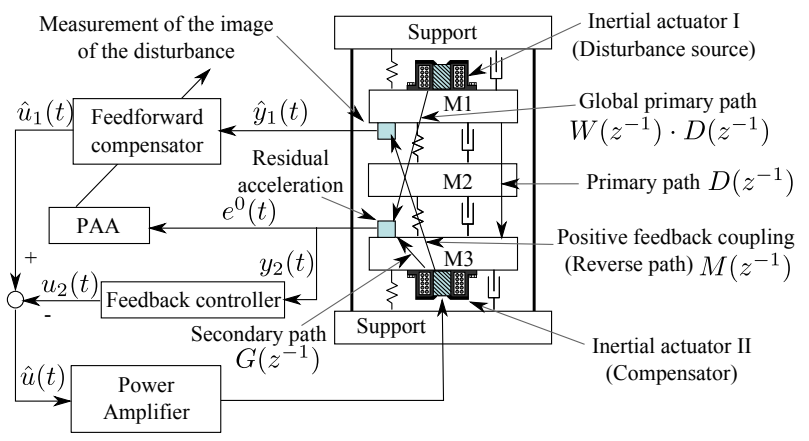

Figure 5. An AVC system using feedforward and feedback compensation - block diagram.

Figs. 4 and 5 show an AVC system applied to a distributed flexible mechanical structure. The corresponding block diagram is shown in Fig. 2. This mechanical structure is representative for a number of situations encountered in practice and will be used to illustrate the performance of the various algorithms which will be presented in this paper.

It consists of five metal plates connected by springs. The uppermost and lowermost ones are rigidly jointed together by four screws. The middle three plates will be labeled for easier referencing M1, M2 and M3 (see Fig. 5). M1 and M3 are equipped with inertial actuators. The one on M1 serves as disturbance generator (inertial actuator I in Fig. 5), the one at the bottom serves for disturbance compensation (inertial actuator II in Fig. 5). Inertial actuators use a similar principle as loudspeakers (see $[26,12])$. The correlated measurement with the disturbance (image of the disturbance) is 
obtained from an accelerometer which is positioned on plate M1. Another sensor of the same type is positioned on plate M3 and serves for measuring the residual acceleration (see Fig. 5). The objective is to minimize the residual acceleration measured on plate M3.

The disturbance is the position of the mobile part of the inertial actuator (see Figs. 4 and 5) located on top of the structure. The input to the compensator system is the position of the mobile part of the inertial actuator located on the bottom of the structure. When the compensator system is active, the actuator acts upon the residual acceleration, but also upon the measurement of the image of the disturbance through the reverse path (a positive feedback coupling). The measured quantity $\hat{y}_{1}(t)$ will be the sum of the correlated disturbance measurement $w(t)$ obtained in the absence of the feedforward compensation (see Fig. 6(a)) and of the effect of the actuator used for compensation. This is illustrated in Fig. 7 by the spectral densities of $y_{1}(t)$ in open loop and when feedforward compensation is active (the effect of the mechanical feedback is significant). The corresponding block diagrams, in open loop operation and with the adaptive compensator system, are shown in Figs. 6(a) and 6(b), respectively.

$$
D=\frac{q^{-d_{D}} B_{D}}{A_{D}}, G=\frac{q^{-d_{G}} B_{G}}{A_{G}}, M=\frac{q^{-d_{M}} B_{M}}{A_{M}}
$$

represent the transfer operators associated with the primary $(D)$, secondary $(G)$ and reverse $(M)$ paths (all asymptotically stable), with

$$
\begin{gathered}
B_{X}\left(q^{-1}\right)=b_{1}^{X} q^{-1}+\ldots+b_{n_{B_{X}}}^{X} q^{-n_{B_{X}}}=q^{-1} B_{X}^{*}\left(q^{-1}\right), \\
A_{X}\left(q^{-1}\right)=1+a_{1}^{X} q^{-1}+\ldots+a_{n_{A_{X}}}^{X} q^{-n_{A_{X}}} .
\end{gathered}
$$

for $X \in D, G, M$ and $d_{X}$ is the plant pure time delay in number of sampling periods ${ }^{3}$.

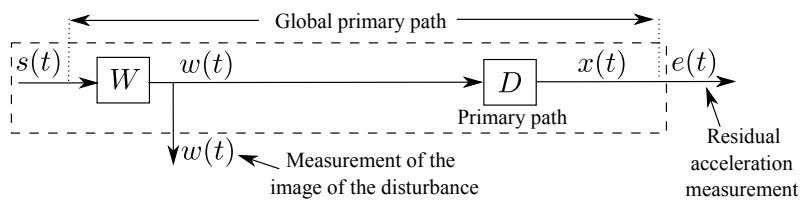

(a)

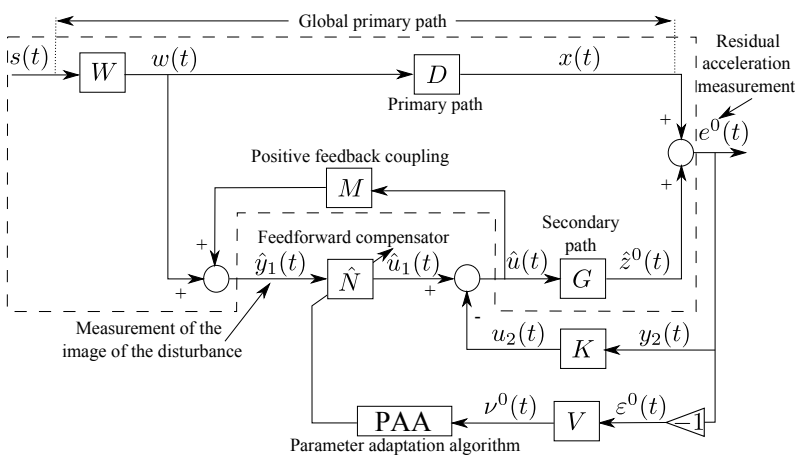

(b)

Figure 6. Feedforward AVC: (a) in open loop and (b) with adaptive feedforward + fixed feedback compensator.

The frequency characteristics of the identified models of the primary path, secondary path and reverse path are shown in Fig. 8. The system shown in Fig. 6(b) can be represented in the standard feedback form shown in Fig. 3.

\footnotetext{
${ }^{3}$ The complex variable $z^{-1}$ will be used to characterize the system's behaviour in the frequency domain and the delay operator $q^{-1}$ will be used for the time domain analysis.
} 


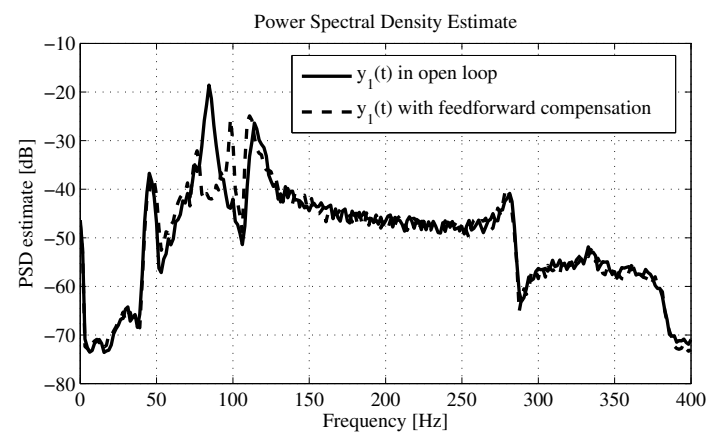

Figure 7. Spectral densities of the image of the disturbance $y_{1}$ in open loop and when feedforward compensation scheme is active (experimental).

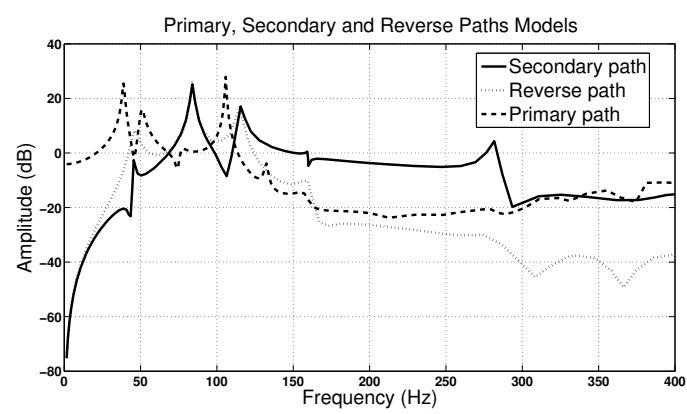

Figure 8. Frequency characteristics of the primary, secondary and reverse paths.

At this stage it is important to make the following remarks:

- very reliable models for the secondary path and the "positive" feedback path can be identified;

- design of a fixed model based stabilizing feedforward compensator requires the knowledge of the reverse path model only;

- knowledge of the disturbance characteristics and of the primary, secondary and reverse paths models is mandatory for the design of an optimal fixed model based feedforward compensator $([1,2])$;

- adaptation algorithms do not use information neither upon the primary path whose characteristics may be unknown nor upon the disturbance characteristics.

\section{ADAPTIVE FEEDBACK ATTENUATION OF MULTIPLE UNKNOWN AND TIME-VARYING NARROW BAND DISTURBANCES}

\subsection{Background}

The objective is to reject asymptotically or strongly attenuate multiple narrow band disturbances which have unknown or time-varying spikes in the frequency domain. To asymptotically reject the disturbance, the Internal Model Principle (IMP) has to be applied. As a consequence, the controller should include a model of the disturbance. Since the disturbances are unknown, two approaches can be considered:

- Indirect adaptive control (one has to identify the model of the disturbance and recompute the controller which will include the estimated model of the disturbance).

- Direct adaptive control (the controller parameters will be directly adapted).

Furthermore for the indirect case one has to construct an observer for the disturbance. An important issue is the tuning of the controller as a function of the model of the disturbance but without 
affecting the stability of the closed loop. It turns out that Youla-Kučera parametrization provides the good parametrization of the controller and provides also a good configuration for obtaining a disturbance observer. It also makes possible to build a direct adaptive regulation scheme where the number of parameters to adapt depends upon the complexity of the disturbance (number of spikes) and not upon the complexity of the secondary path model. However since the Youla-Kučera parametrization is not unique different adaptive controller configurations will be obtained depending on the parametrization used [25].

\subsection{Controller structure}

The structure of the LTI discrete time model of the plant, also called secondary path $(\mathrm{G})$, used for controller design is given in (3), (4) and (5). For simplifying the writing one takes $d_{G}=d$. The

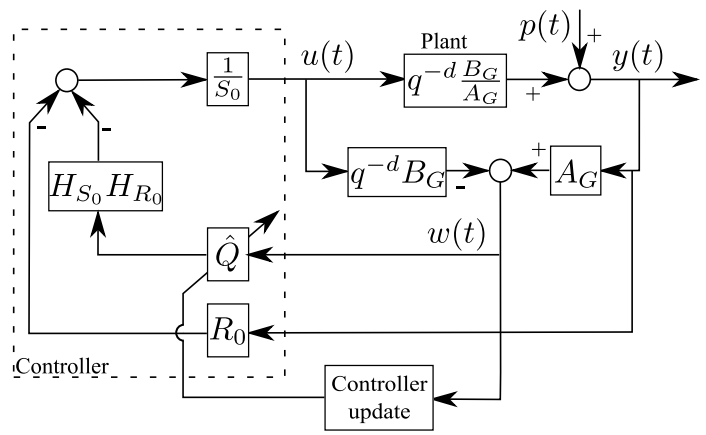

Figure 9. Direct adaptive regulation scheme for rejection of unknown disturbances.

output of the plant $y(t)$ and the input $u(t)$ in the absence of the Youla-Kučera filters, may be written as (consider Fig. 9 without the filter $\hat{Q}$ ):

$$
\begin{aligned}
y(t) & =\frac{q^{-d} B_{G}\left(q^{-1}\right)}{A_{G}\left(q^{-1}\right)} \cdot u(t)+p(t), \\
S_{0}\left(q^{-1}\right) \cdot u(t) & =-R_{0}\left(q^{-1}\right) \cdot y(t) .
\end{aligned}
$$

In (6), $p(t)$ is the effect of the disturbances on the measured output ${ }^{4}$ and $R_{0}\left(z^{-1}\right), S_{0}\left(z^{-1}\right)$ are polynomials in $z^{-1}$ having the following expressions ${ }^{5}$ :

$$
\begin{aligned}
& S_{0}=1+s_{1}^{0} z^{-1}+\ldots+s_{n_{S_{0}}}^{0} z^{-n_{S_{0}}}=S_{0}^{\prime}\left(z^{-1}\right) \cdot H_{S_{0}}\left(z^{-1}\right), \\
& R_{0}=r_{0}^{0}+r_{1}^{0} z^{-1}+\ldots+r_{n_{R_{0}}}^{0} z^{-n_{R_{0}}}=R_{0}^{\prime}\left(z^{-1}\right) \cdot H_{R_{0}}\left(z^{-1}\right),
\end{aligned}
$$

where $H_{S_{0}}\left(q^{-1}\right)$ and $H_{R_{0}}\left(q^{-1}\right)$ represent pre-specified parts of the controller (used for example to incorporate the internal model of a disturbance or to open the loop at certain frequencies) and $S_{0}^{\prime}\left(q^{-1}\right)$ and $R_{0}^{\prime}\left(q^{-1}\right)$ are computed. The characteristic polynomial, which specifies the desired closed loop poles of the system is given by: ${ }^{6}$ (see also [11]).

$$
P_{0}\left(z^{-1}\right)=A_{G}\left(z^{-1}\right) S_{0}\left(z^{-1}\right)+z^{-d} B_{G}\left(z^{-1}\right) R_{0}\left(z^{-1}\right),
$$

In what follows the Youla-Kučera parametrization $([27,28])$ is used. However the Youla-Kučera parametrization is not unique, it depends on the right coprime factorization selected $G=N D^{-1}$. Four factorization are mostly used [25]:

$$
N=G ; \quad D=I .
$$

\footnotetext{
${ }^{4}$ The disturbance passes through a so called primary path which is not represented in this figure, and $p(t)$ is its output.

${ }^{5}$ The argument $\left(z^{-1}\right)$ will be omitted in some of the following equations to make them more compact.

${ }^{6}$ It is assumed that a reliable model identification is achieved and therefore the estimated model is assumed to be equal to the true model.
} 


$$
\begin{gathered}
N=z^{-m} ; \quad D=P_{m} \quad \text { with } \quad G \approx z^{-m} P_{m}^{-1} . \\
N=q^{-d} B_{G} ; \quad D=A_{G} \quad \text { with } \quad G=q^{-d} \frac{B_{G}}{A_{G}} . \\
N=q^{-d} B_{G} F ; D=A_{G} F \quad \text { with } \quad G=q^{-d} \frac{B_{G}}{A_{G}} ; F=\frac{F_{N}}{F_{D}},
\end{gathered}
$$

with $F$ and $F^{-1}$ asymptotically stable. More details can be found in [25]. We will consider subsequently the parametrization (13).

Selecting a FIR structure for the Q filter associated to the Youla-Kučera parametrization, the controller's polynomials become:

$$
\begin{aligned}
& R=R_{0}+A_{G} Q H_{S_{0}} H_{R_{0}}, \\
& S=S_{0}-z^{-d} B_{G} Q H_{S_{0}} H_{R_{0}},
\end{aligned}
$$

where $R_{0}$ and $S_{0}$ define the central controller which verifies the desired specifications in the absence of the disturbance. The characteristic polynomial of the closed loop is still given by (10). We define the output sensitivity function (the transfer function between the disturbance $p(t)$ and the output of the system $y(t))$ as

$$
S_{y p}\left(z^{-1}\right)=\frac{A_{G}\left(z^{-1}\right) S\left(z^{-1}\right)}{P_{0}\left(z^{-1}\right)}
$$

and the input sensitivity function (the transfer function between the disturbance $p(t)$ and the control input $u(t))$ as

$$
S_{u p}\left(z^{-1}\right)=-\frac{A_{G}\left(z^{-1}\right) R\left(z^{-1}\right)}{P_{0}\left(z^{-1}\right)},
$$

\subsection{Direct adaptive regulation using Youla-Kučera parametrization}

A key aspect of this methodology is the use of the Internal Model Principle (IMP). It is supposed that $p(t)$ is a deterministic disturbance given by

$$
p(t)=\frac{N_{p}\left(q^{-1}\right)}{D_{p}\left(q^{-1}\right)} \cdot \delta(t)
$$

where $\delta(t)$ is a Dirac impulse and $N_{p}, D_{p}$ are coprime polynomials of degrees $n_{N_{p}}$ and $n_{D_{p}}$, respectively ${ }^{7}$. In the case of stationary narrow-band disturbances, the roots of $D_{p}\left(z^{-1}\right)$ are on the unit circle.

Internal Model Principle [10]: The effect of the disturbance given in (19) upon the output is given by

$$
y(t)=\frac{A_{G}\left(q^{-1}\right) S\left(q^{-1}\right)}{P\left(q^{-1}\right)} \cdot \frac{N_{p}\left(q^{-1}\right)}{D_{p}\left(q^{-1}\right)} \cdot \delta(t),
$$

where $D_{p}\left(z^{-1}\right)$ is a polynomial with roots on the unit circle and $P\left(z^{-1}\right)$ is an asymptotically stable polynomial. $y(t)$ in 20 converges asymptotically towards zero iff the polynomial $S\left(z^{-1}\right)$ in the RS controller has the form (based on eq. (8))

$$
S\left(z^{-1}\right)=D_{p}\left(z^{-1}\right) H_{S_{0}}\left(z^{-1}\right) S^{\prime}\left(z^{-1}\right) .
$$

Thus, the pre-specified part of $S\left(z^{-1}\right)$ should be chosen as $H_{S}\left(z^{-1}\right)=D_{p}\left(z^{-1}\right) H_{S_{0}}\left(z^{-1}\right)$ and the controller is computed solving

$$
P=A_{G} D_{p} H_{S_{0}} S^{\prime}+z^{-d} B_{G} H_{R_{0}} R^{\prime},
$$

\footnotetext{
${ }^{7}$ Throughout the paper, $n_{X}$ denotes the degree of the polynomial $X$.
} 
where $P, D_{p}, A_{G}, B_{G}, H_{R_{0}}, H_{S_{0}}$ and $d$ are given ${ }^{8}$.

For the purpose of direct adaptive regulation, $Q\left(z^{-1}\right)$ is considered to be a FIR filter $\left(A_{Q}\left(z^{-1}\right)=\right.$ 1 and $Q\left(z^{-1}\right)=B_{Q}\left(z^{-1}\right)$ )

$$
Q\left(z^{-1}\right)=q_{0}+q_{1} z^{-1}+\ldots+q_{n_{Q}} z^{-n_{Q}} .
$$

To compute $Q\left(z^{-1}\right)$ in order that the polynomial $S\left(z^{-1}\right)$ given by (16) incorporates the internal model of the disturbance (21), one has to solve the diophantine equation

$$
S^{\prime} D_{p}+z^{-d} B_{G} H_{R_{0}} Q=S_{0}^{\prime},
$$

where $D_{p}, d, B_{G}, S_{0}^{\prime}$, and $H_{R_{0}}$ are known and $S^{\prime}$ and $Q$ are unknown. Eq. (24) has a unique solution for $S^{\prime}$ and $Q$ with: $n_{S_{0}^{\prime}} \leq n_{D_{p}}+n_{B_{G}}+d+n_{H_{R_{0}}}-1, n_{S^{\prime}}=n_{B_{G}}+d+n_{H_{R_{0}}}-1$, $n_{Q}=n_{D_{p}}-1$. One sees that the order $n_{Q}$ of the polynomial $Q$ depends upon the structure of the disturbance model. The use of the Youla-Kučera parametrization, with $Q$ given in (23), is interesting in this case because it allows to maintain the closed loop poles as given by the central controller but at the same time introduces the parameters of the internal model into the controller. To build the parametric adaptation algorithm (PAA), one has to find first an error equation (see also $[28,19,29])$. Using the Q-parametrization, the output of the system in the presence of a disturbance can be expressed as

$$
y(t)=\frac{A_{G}\left[S_{0}-q^{-d} B_{G} H_{S_{0}} H_{R_{0}} Q\right]}{P} \cdot \frac{N_{p}}{D_{p}} \cdot \delta(t)=\frac{S_{0}-q^{-d} B_{G} H_{S_{0}} H_{R_{0}} Q}{P} \cdot w(t),
$$

where $w(t)$ is given by (see also Fig. 9)

$$
w(t)=\frac{A_{G} N_{p}}{D_{p}} \cdot \delta(t)=A_{G} \cdot y(t)-q^{-d} \cdot B_{G} \cdot u(t) .
$$

Taking into consideration that the adaptation of $Q$ is done in order to obtain an output $y(t)$ which tends asymptotically to zero, one can define $\varepsilon^{0}(t+1)$ as the value of $y(t+1)$ obtained with $\hat{Q}\left(t, q^{-1}\right)$ (the estimate of $Q$ at time $t$, written also $\left.\hat{Q}(t)\right)$

$$
\varepsilon^{0}(t+1)=\frac{S_{0}}{P} \cdot w(t+1)-\hat{Q}(t) \frac{q^{-d} B_{G}^{*} H_{S_{0}} H_{R_{0}}}{P} \cdot w(t) .
$$

Similarly, the a posteriori error becomes (using $\hat{Q}(t+1))$ as $^{9}$.

$$
\varepsilon(t+1)=\frac{S_{0}}{P} \cdot w(t+1)-\hat{Q}(t+1) \frac{q^{-d} B_{G}^{*} H_{S_{0}} H_{R_{0}}}{P} \cdot w(t) .
$$

Replacing $S_{0}$ from the last equation by (24), one obtains

$$
\varepsilon(t+1)=[Q-\hat{Q}(t+1)] \cdot \frac{q^{-d} B_{G}^{*} H_{S_{0}} H_{R_{0}}}{P} \cdot w(t)+v(t+1),
$$

where

$$
v(t)=\frac{S^{\prime} D_{p} H_{S_{0}}}{P} \cdot w(t)=\frac{S^{\prime} H_{S_{0}} A_{G} N_{p}}{P} \cdot \delta(t)
$$

is a signal which tends asymptotically towards zero. Define the estimated polynomial $\hat{Q}\left(t, q^{-1}\right)=$ $\hat{q}_{0}(t)+\hat{q}_{1}(t) q^{-1}+\ldots+\hat{q}_{n_{Q}}(t) q^{-n_{Q}}$ and the associated estimated parameter vector $\hat{\theta}(t)=$

${ }^{8}$ Of course, it is assumed that $D_{p}$ and $B_{G}$ do not have common factors.

${ }^{9}$ In adaptive control and estimation the predicted output at $t+1$ can be computed either on the basis of the previous parameter estimates (a priori, time $t$ ) or on the basis of the current parameter estimates (a posteriori, time $t+1$ ). 


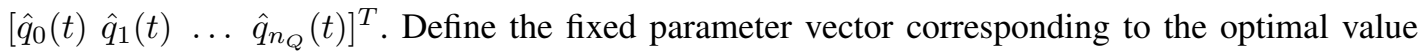
of the polynomial $Q$ as: $\theta=\left[\begin{array}{llll}q_{0} & q_{1} & \ldots & q_{n_{Q}}\end{array}\right]^{T}$.

Denote

$$
w_{2}(t)=\frac{q^{-d} B_{G}^{*} H_{S_{0}} H_{R_{0}}}{P} \cdot w(t)
$$

and define the following observation vector

$$
\phi^{T}(t)=\left[w_{2}(t) w_{2}(t-1) \ldots w_{2}\left(t-n_{Q}\right)\right]
$$

Eq. (29) becomes

$$
\varepsilon(t+1)=\left[\theta^{T}-\hat{\theta}^{T}(t+1)\right] \cdot \phi(t)+v(t+1) .
$$

One can remark that $\varepsilon(t+1)$ corresponds to an a posteriori adaptation error ([10]).

From eq. (27), one obtains the a priori adaptation error

$$
\varepsilon^{0}(t+1)=w_{1}(t+1)-\hat{\theta}^{T}(t) \phi(t)
$$

with

$$
\begin{aligned}
w_{1}(t+1) & =\frac{S_{0}\left(q^{-1}\right)}{P\left(q^{-1}\right)} \cdot w(t+1), \\
w(t+1) & =A_{G}\left(q^{-1}\right) \cdot y(t+1)-q^{-d} B_{G}^{*}\left(q^{-1}\right) \cdot u(t),
\end{aligned}
$$

where $B_{G}\left(q^{-1}\right) u(t+1)=B_{G}^{*}\left(q^{-1}\right) u(t)$.

The a posteriori adaptation error is obtained from (28)

$$
\varepsilon(t+1)=w_{1}(t+1)-\hat{\theta}^{T}(t+1) \phi(t) .
$$

For the estimation of the parameters of $\hat{Q}\left(t, q^{-1}\right)$ the following PAA is used ([10]):

$$
\begin{aligned}
\hat{\theta}(t+1) & =\hat{\theta}(t)+F(t) \phi(t) \varepsilon(t+1), \\
\varepsilon(t+1) & =\frac{\varepsilon^{0}(t+1)}{1+\phi^{T}(t) F(t) \phi(t)}, \\
\varepsilon^{0}(t+1) & =w_{1}(t+1)-\hat{\theta}^{T}(t) \phi(t), \\
F(t+1) & =\frac{1}{\lambda_{1}(t)}\left[F(t)-\frac{F(t) \phi(t) \phi^{T}(t) F(t)}{\frac{\lambda_{1}(t)}{\lambda_{2}(t)}+\phi^{T}(t) F(t) \phi(t)}\right], \\
1 & \geq \lambda_{1}(t)>0,0 \leq \lambda_{2}(t)<2,
\end{aligned}
$$

where $\lambda_{1}(t), \lambda_{2}(t)$ allow to obtain various profiles for the evolution of the adaption gain $F(t)$ (for details see $[10,11])$. For a stability proof under the hypothesis model=plant ${ }^{10}$ see [19]

\subsection{Robustness considerations}

To avoid unacceptable high values of the modulus of the output sensitivity function when internal model principle is used, a robust control design should be considered assuming that the model of the disturbance and its domain of variation in the frequency domain are known. The objective is that for all situations, acceptable modulus margin $\left(\left|S_{y p}\left(e^{-j \omega}\right)\right|_{\text {max }}^{-1}\right)$ and delay margin are obtained.

Furthermore, at the frequencies where perfect rejection of the disturbance is achieved one has $S_{y p}\left(e^{-j \omega}\right)=0$ and

$$
\left|S_{u p}\left(e^{-j \omega}\right)\right|=\left|\frac{A_{G}\left(e^{-j \omega}\right)}{B_{G}\left(e^{-j \omega}\right)}\right| .
$$

\footnotetext{
$\overline{{ }^{10} \text { No positive real }}$ condition required for asymptotic stability.
} 
Equation (43) corresponds to the inverse of the gain of the system to be controlled. The implication of equation (43) is that cancelation (or in general an important attenuation) of disturbances on the output should be done only in frequency regions where the system gain is large enough. If the gain of the controlled system is too low, $\left|S_{u p}\right|$ will be large at these frequencies. Therefore, the robustness versus additive plant model uncertainties will be reduced and the stress on the actuator will become important [12].

\subsection{Indirect Adaptive Regulation Based on Shaping of the Output Sensitivity Function}

The purpose of this method is to allow the possibility of choosing the desired attenuation and bandwidth of attenuation for each of the estimated narrow-band disturbances and as a consequence to reduce the "water bed" effect on the output sensitivity function with respect to the IMP approach. This is achieved by the shaping of the output sensitivity function.

The design uses Band Stop Filters (BSFs) to shape the output sensitivity function. Following $[11,30]$, there exist digital filters ${ }^{11} \frac{H_{S_{i}}}{P_{F_{i}}}$, which will assure the desired attenuation of a narrow-band disturbance ( $i \in\{1, \ldots, n\}$ and $n=\frac{n_{D_{p}}}{2}$ from the previous section). The structure of the BSFs is

$$
\frac{S_{B S F_{i}}\left(z^{-1}\right)}{P_{B S F_{i}}\left(z^{-1}\right)}=\frac{1+\beta_{1}^{i} z^{-1}+\beta_{2}^{i} z^{-1}}{1+\alpha_{1}^{i} z^{-1}+\alpha_{2}^{i} z^{-1}},
$$

resulting from the discretization of a continuous filter (see also [30, 11])

$$
F_{i}(s)=\frac{s^{2}+2 \zeta_{n_{i}} \omega_{i} s+\omega_{i}^{2}}{s^{2}+2 \zeta_{d_{i}} \omega_{i} s+\omega_{i}^{2}}
$$

using the bilinear transformation. This filter introduces an attenuation of

$$
M_{i}=-20 \cdot \log _{10}\left(\frac{\zeta_{n_{i}}}{\zeta_{d_{i}}}\right)
$$

at the frequency $\omega_{i}$. Positive values of $M_{i}$ denote attenuations $\left(\zeta_{n_{i}}<\zeta_{d_{i}}\right)$.

Under the hypothesis that the plant model parameters are constant and that an accurate identification experiment can be run, a reliable estimate $\hat{p}(t)$ of the disturbance signal can be obtain by using the disturbance observer

$$
\hat{p}(t+1)=y(t+1)-q^{-d} \frac{B_{G}^{*}\left(q^{-1}\right)}{A_{G}\left(q^{-1}\right)} u(t) .
$$

The signal $\hat{p}(t)$ can then be used to estimate the spike frequencies $\left(\hat{\omega}_{i}\right)$ with adaptive notch filters (ANF) as described in [31, 32].

Remark: The design parameters for each BSF are the desired attenuation $\left(M_{i}\right)$, the central frequency of the filter $\left(\hat{\omega}_{i}\right)$ and the damping of the denominator $\left(\zeta_{d_{i}}\right)$. The denominator damping is used to adjust the frequency bandwidth of the BSF.

For $n$ narrow-band disturbances, $n$ BSFs will be used

$$
H_{B S F}\left(z^{-1}\right)=\frac{S_{B S F}\left(z^{-1}\right)}{P_{B S F}\left(z^{-1}\right)}=\frac{\prod_{i=1}^{n} S_{B S F_{i}}\left(z^{-1}\right)}{\prod_{i=1}^{n} P_{B S F_{i}}\left(z^{-1}\right)} .
$$

$S\left(z^{-1}\right)$ and $R\left(z^{-1}\right)$ are obtained as solutions of the Bezout equation

$$
P\left(z^{-1}\right)=A_{G}\left(z^{-1}\right) S\left(z^{-1}\right)+z^{-d} B_{G}\left(z^{-1}\right) R\left(z^{-1}\right),
$$

\footnotetext{
${ }^{11}$ The numerators of these filters will be implemented in the controller while the denominators will define additional closed loop poles.
} 
where

$$
R\left(z^{-1}\right)=H_{R}\left(z^{-1}\right) R^{\prime}\left(z^{-1}\right), S\left(z^{-1}\right)=H_{S}\left(z^{-1}\right) S^{\prime}\left(z^{-1}\right),
$$

and $P\left(z^{-1}\right)$ is given by

$$
P\left(z^{-1}\right)=P_{0}\left(z^{-1}\right) P_{B S F}\left(z^{-1}\right) .
$$

In the last equation, $P_{B S F}$ is the combined denominator of all the BSFs, (48), and $P_{0}$ was defined in (10). The fixed part of the controller denominator $H_{S}$ is in turn factorized into

$$
H_{S}\left(z^{-1}\right)=S_{B S F}\left(z^{-1}\right) H_{S_{0}}\left(z^{-1}\right)
$$

where $S_{B S F}$ is the combined numerator of the BSF, (48), and $H_{S_{0}}$ has been introduced in (8). It is easy to see that the output sensitivity function becomes

$$
S_{y p}\left(z^{-1}\right)=\frac{A_{G}\left(z^{-1}\right) S^{\prime}\left(z^{-1}\right) H_{S_{0}}\left(z^{-1}\right) S_{B S F}\left(z^{-1}\right)}{P_{0}\left(z^{-1}\right) P_{B S F}\left(z^{-1}\right)}
$$

and the shaping effect of the BSFs upon the sensitivity functions is obvious.

The unknowns $S^{\prime}$ and $R^{\prime}$ are solutions of

$$
\begin{aligned}
P\left(z^{-1}\right) & =P_{0}\left(z^{-1}\right) P_{B S F}\left(z^{-1}\right) \\
& =A_{G}\left(z^{-1}\right) H_{S}\left(z^{-1}\right) S^{\prime}\left(z^{-1}\right)+z^{-d} B_{G}\left(z^{-1}\right) H_{R_{0}}\left(z^{-1}\right) R^{\prime}\left(z^{-1}\right)
\end{aligned}
$$

and can be computed by putting (54) into matrix form (see also [11]). The size of the matrix equation that needs to be solved is given by

$$
n_{B e z}=n_{A_{G}}+n_{B_{G}}+d+n_{H_{S_{0}}}+n_{H_{R_{0}}}+2 \cdot n-1,
$$

where $n_{A_{G}}, n_{B_{G}}$, and $d$ are respectively the order of the plant's model denominator, numerator, and delay, $n_{H_{S_{0}}}$ and $n_{H_{R_{0}}}$ are the orders of $H_{S_{0}}\left(z^{-1}\right)$ and $H_{R_{0}}\left(z^{-1}\right)$ respectively and $n$ is the number of narrow-band disturbances.

The computational complexity related to the Bezout equation (54) is significant. However, using the Youla-Kučera parametrization an important reduction of the computation load can be obtained. Details can be found in [33].

In order to use the proposed control strategy in the presence of unknown and/or time-varying narrow-band disturbances, one needs an estimation in real time of the spikes' frequencies in the spectrum of the disturbance. In the framework of narrow-band disturbance rejection, it is usually supposed that the disturbances are in fact sinusoidal signals with variable frequencies. An estimator of the spikes frequency can then be used. However in order to do this an observer for the disturbance has to be built as indicated in eq. (47). Combining the control procedure presented above with a spikes frequency estimator an indirect adaptive regulation scheme is obtained. A stability analysis of the full scheme is available in [34].

\section{SOME EXPERIMENTAL RESULTS - ATTENUATION OF MULTIPLE NARROW BAND DISTURBANCES}

Performance of some algorithms for adaptive attenuation of unknown and time-varying disturbances will be illustrated on the experimental platform described in Section 2.

Adaptive feedback attenuation of unknown and time-varying multiple narrow band disturbances Attenuation of unknown and time-varying multiple narrow band disturbances by adaptive feedback will be illustrated next using the direct adaptive approach using IMP presented in Subsection 3.3 and the indirect adaptive approach using shaping of the output sensitivity function presented in Subsection 3.5. Two tests were considered for the case of 3 sinusoidal disturbances: i) a constant disturbance frequency and ii) a variable disturbance frequency. The first one is used for a steady state 


\begin{tabular}{ccccccc}
\hline & \multicolumn{9}{l}{ Three sinusoids: $55-75-95 \mathrm{~Hz}$} \\
\hline Measurement & $\mathbf{G A}$ & \multicolumn{3}{c}{$\mathbf{D A}$} & \multicolumn{3}{c}{$\mathbf{M A}$} \\
\cline { 2 - 7 } Units & $\mathbf{d B}$ & $\mathbf{d B}$ & $\mathbf{d B}$ & $\mathbf{d B}$ & $\mathbf{d B}$ & $\mathbf{H z}$ \\
\cline { 2 - 7 } Direct & 41.33 & 45.01 & 47.98 & 45.25 & 14.39 & 164.10 \\
\cline { 2 - 7 } Indirect & 45.40 & 56.35 & 45.06 & 57.72 & 11.72 & 65.63 \\
\hline
\end{tabular}

Table I. Constant Disturbance Frequency - Adaptive feedback attenuation

performance comparison and the second for a transient performance comparison. Table I shows the results obtained once the adaptation is settled, for a constant disturbance frequency. Global Attenuation (GA), Disturbance Attenuation (DA), Maximum Amplification (MA) are evaluated. The indirect adaptive approach shows better global attenuation and disturbance attenuation with lower maximum amplification. In Fig. 10, the power spectral density (PSD) estimates for 55, 75 and $95 \mathrm{~Hz}$ disturbance are shown (computed after the adaptation process has converged towards an almost constant controller). The indirect adaptive algorithm of [34] introduces a larger attenuation of the spikes, having a lower maximum amplification as the direct adaptive algorithm, this is due to the introduction of low damped complex poles in order to minimize the "water bed" effect over the output sensitivity function. In Fig. 11, the effects of three sequences of multi-sinusoidal disturbances applied to the primary path are shown. Their corresponding frequencies are indicated in each of the figures. The signal on top represent the effect of the disturbance upon the residual acceleration in open loop operation when a variable disturbance frequency is used, the one in the middle correspond to the residual acceleration in closed loop with the indirect adaptive algorithm using BSF algorithm and the one on the bottom are the residual accelerations obtained with the direct adaptive IMP algorithm. The transients duration for IMP is slightly shorter than for BSF.

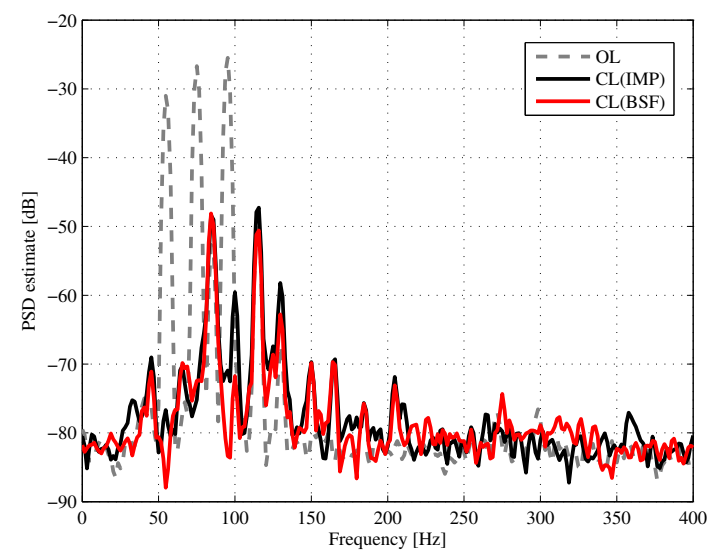

Figure 10. PSD comparison between the open loop measured disturbance and the residual accelerations obtained with direct and indirect adaptation .

The performances of the two schemes depend also on how the central controller is designed. The indirect approach is less sensitive with respect to the design of the central controller. Further details concerning the design of the central controller for this these two schemes can be found in $[35,33]$. It has to ne noted that the computation load is significantly more important for the indirect approach than for the direct approach. For further comparison of the two approaches in the context of a benchmark problem (an active suspension system), see [25]. 

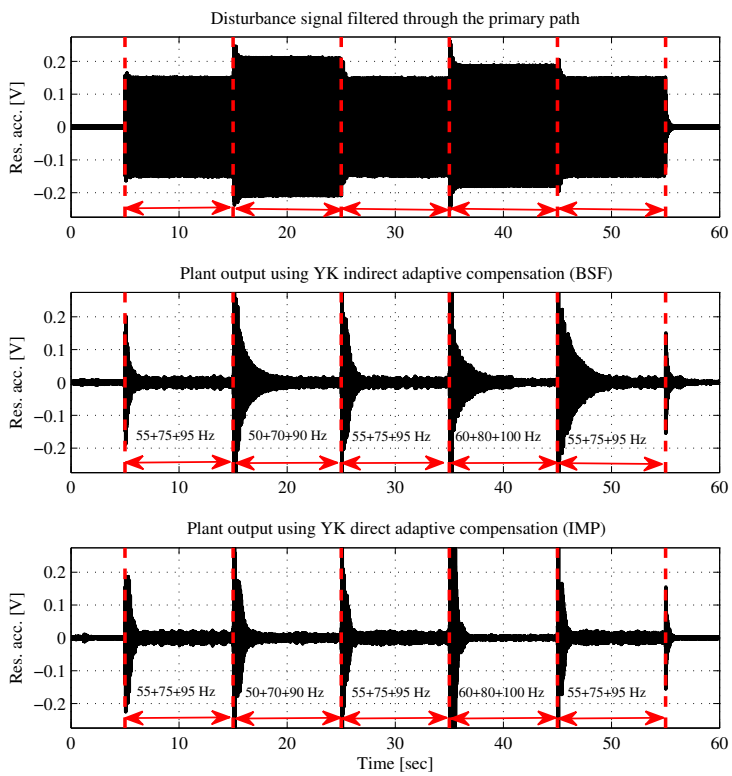

Figure 11. Performance comparison in the presence of a three sinusoidal disturbances.

\section{ADAPTIVE FEEDFORWARD + FIXED FEEDBACK COMPENSATION OF BROAD BAND DISTURBANCES}

For broad band disturbance attenuation the use of the feedforward compensation is mandatory (as explained in the Introduction). However adding feedback should improve the global performance. Of course the algorithms for the hybrid feedforward+feedback control can be particularized for the case without feedback control.

\subsection{Basic Equations and Notations}

The block diagram associated with an AVC system using an hybrid (feedback + adaptive feedforward) control is shown in Fig. 6. The transfer operators characterizing the primary path (D), the secondary path (G) and the reverse path (M) are given in (3), (4) and (5). To simplify the writing, the delay $d_{X}$ is incorporated in $B_{X}$ where $X \in D, G, M$.

The optimal feedforward filter (unknown) is defined by

$$
N\left(q^{-1}\right)=\frac{R\left(q^{-1}\right)}{S\left(q^{-1}\right)}
$$

where

$$
\begin{gathered}
R\left(q^{-1}\right)=r_{0}+r_{1} q^{-1}+\ldots+r_{n_{R}} q^{-n_{R}}, \\
S\left(q^{-1}\right)=1+s_{1} q^{-1}+\ldots+s_{n_{S}} q^{-n_{S}}=1+q^{-1} S^{*}\left(q^{-1}\right) .
\end{gathered}
$$

The estimated feedforward filter is denoted by

$$
\hat{N}\left(q^{-1}\right)=\frac{\hat{R}\left(q^{-1}\right)}{\hat{S}\left(q^{-1}\right)} .
$$

The vector of optimal feedforward filter parameters is

$$
\theta^{T}=\left[s_{1}, \ldots s_{n_{S}}, r_{0}, \ldots r_{n_{R}}\right]^{T}
$$


and the vector of estimated feedforward filter coefficients is

$$
\hat{\theta}^{T}(t)=\left[\hat{s}_{1}(t), \ldots \hat{s}_{n_{S}}(t), \hat{r}_{0}(t), \ldots \hat{r}_{n_{R}}(t)\right]^{T} .
$$

The fixed RS controller $K$, computed on the basis of the model $\hat{G}$ to reject broadband disturbances on the output $e(t)$, is characterized by the asymptotically stable transfer function

$$
K\left(q^{-1}\right)=\frac{B_{K}\left(q^{-1}\right)}{A_{K}\left(q^{-1}\right)},
$$

where

$$
\begin{gathered}
B_{K}\left(q^{-1}\right)=b_{0}^{K}+b_{1}^{K} q^{-1}+\ldots+b_{n_{B_{K}}^{K}}^{K} q^{-n_{B_{K}}}, \\
A_{K}\left(q^{-1}\right)=1+a_{1}^{K} q^{-1}+\ldots+a_{n_{A_{K}}}^{K} q^{-n_{A_{K}} .}
\end{gathered}
$$

The input of the feedforward filter (called also reference) is denoted by $\hat{y}_{1}(t)$ and it corresponds to the measurement provided by the primary transducer (force or acceleration transducer in AVC or a microphone in ANC). The output of the feedforward compensator is denoted by $\hat{u}_{1}(t+1)=$ $\hat{u}_{1}(t+1 \mid \hat{\theta}(t+1))$ (a posteriori output). The measured input to the feedforward filter can also be written as

$$
\hat{y}_{1}(t+1)=w(t+1)+\frac{B_{M}^{*}\left(q^{-1}\right)}{A_{M}\left(q^{-1}\right)} \hat{u}(t),
$$

where

$$
\hat{u}=\hat{u}_{1}(t)-u_{2}(t),
$$

$\hat{u}_{1}(t)$ and $u_{2}(t)$ are the outputs given by the adaptive feedforward and the fixed feedback compensator, respectively. $\hat{u}$ is the effective input sent to the control actuator.

The $a$ priori output of the estimated feedforward filter is given by

$$
\begin{aligned}
\hat{u}_{1}^{0}(t+1) & =\hat{u}_{1}(t+1 \mid \hat{\theta}(t))=-\hat{S}^{*}\left(t, q^{-1}\right) \hat{u}_{1}(t)+\hat{R}\left(t, q^{-1}\right) \hat{y}_{1}(t+1) \\
& =\hat{\theta}^{T}(t) \phi(t)=\left[\hat{\theta}_{S}^{T}(t), \hat{\theta}_{R}^{T}(t)\right]\left[\begin{array}{c}
\phi_{\hat{u}_{1}}(t) \\
\phi_{\hat{y}_{1}}(t)
\end{array}\right]
\end{aligned}
$$

where $\hat{\theta}^{T}(t)$ has been given in (6) and

$$
\phi^{T}(t)=\left[-\hat{u}_{1}(t), \ldots-\hat{u}_{1}\left(t-n_{S}+1\right), \hat{y}_{1}(t+1), \ldots \hat{y}_{1}\left(t-n_{R}+1\right)\right]=\left[\phi_{\hat{u}_{1}}^{T}(t), \phi_{\hat{y}_{1}}^{T}(t)\right]
$$

The input to the feedback (fixed) compensator is given by the performance variable, therefore $y_{2}(t)=e(t)$. Its output will be $u_{2}(t)=K \cdot y_{2}(t)$. The unmeasurable value of the output of the primary path (when the compensation is active) is denoted $x(t)$. The a priori output of the secondary path is denoted $\hat{z}^{0}(t+1)=\hat{z}(t+1 \mid \hat{\theta}(t))$ while its input is $\hat{u}(t)$. One has

$$
\hat{z}^{0}(t+1)=\frac{B_{G}^{*}\left(q^{-1}\right)}{A_{G}\left(q^{-1}\right)} \hat{u}(t)=\frac{B_{G}^{*}\left(q^{-1}\right)}{A_{G}\left(q^{-1}\right)} \hat{u}(t \mid \hat{\theta}(t)) .
$$

The measured residual acceleration (or force) satisfies the following equation

$$
e^{0}(t+1)=x(t+1)+\hat{z}^{0}(t+1) .
$$

The $a$ priori and a posteriori adaptation error are defined as

$$
\varepsilon^{0}(t+1)=\varepsilon(t+1 \mid \hat{\theta}(t))=-e^{0}(t+1)
$$

and

$$
\varepsilon(t+1)=\varepsilon(t+1 \mid \hat{\theta}(t+1))=-e(t+1)=-x(t+1)-\hat{z}(t+1)
$$

where the a posteriori value of the output of the secondary path $\hat{z}(t+1)$ (dummy variable) is given by

$$
\hat{z}(t+1)=\hat{z}(t+1 \mid \hat{\theta}(t+1))=\frac{B_{G}^{*}\left(q^{-1}\right)}{A_{G}\left(q^{-1}\right)} \hat{u}(t \mid \hat{\theta}(t+1)) .
$$

For compensators with constant parameters $\varepsilon^{0}(t)=\varepsilon(t), e^{0}(t)=e(t), \hat{z}^{0}(t)=\hat{z}(t), \hat{u}^{0}(t)=\hat{u}(t)$. 


\subsection{Development of the Algorithms}

The algorithms for adaptive feedforward compensation in presence of feedback controller have been developed under the following hypotheses [36]:

H1) The signal $w(t)$ is bounded, i.e.,

$$
|w(t)| \leq \alpha, \quad \forall t \quad(0 \leq \alpha<\infty)
$$

H2) Perfect matching condition - There exists a filter $N\left(q^{-1}\right)$ of finite dimension such that

$$
\frac{N\left(z^{-1}\right)}{1-N\left(z^{-1}\right) M\left(z^{-1}\right)} G\left(z^{-1}\right)=-D\left(z^{-1}\right)
$$

and the characteristic polynomials:

- of the "internal" positive coupling loop

$$
P\left(z^{-1}\right)=A_{M}\left(z^{-1}\right) S\left(z^{-1}\right)-B_{M}\left(z^{-1}\right) R\left(z^{-1}\right),
$$

- of the closed loop (G-K)

$$
P_{c l}\left(z^{-1}\right)=A_{G}\left(z^{-1}\right) A_{K}\left(z^{-1}\right)+B_{G}\left(z^{-1}\right) B_{K}\left(z^{-1}\right),
$$

- and of the coupled feedforward-feedback loop

$$
P_{f b-f f}=A_{M} S\left[A_{G} A_{K}+B_{G} B_{K}\right]-B_{M} R A_{K} A_{G}
$$

are Hurwitz polynomials.

H3) Deterministic context - The effect of the measurement noise upon the measured residual error is neglected.

H4) The primary path model $D\left(z^{-1}\right)$ is unknown and constant.

Under hypotheses $H 1, H 2, H 3$ and $H 4$, for the system described in subsection 5.1 using a feedforward compensator $\hat{N}$ with constant parameters and a feedback controller K, one obtains the following expression for the residual acceleration [37]:

$$
\varepsilon(t+1)=\frac{A_{M} A_{G} A_{K} G}{P_{f b-f f}}[\theta-\hat{\theta}]^{T} \phi(t)
$$

where

$$
\theta^{T}=\left[s_{1}, \ldots s_{n_{S}}, r_{0}, r_{1}, \ldots r_{n_{R}}\right]=\left[\theta_{S}^{T}, \theta_{R}^{T}\right]
$$

is the vector of parameters of the optimal filter $N$ assuring perfect matching,

$$
\hat{\theta}^{T}=\left[\hat{s}_{1} \ldots \hat{s}_{n_{S}}, \hat{r}_{0} \ldots \hat{r}_{n_{R}}\right]=\left[\hat{\theta}_{S}^{T}, \hat{\theta}_{R}^{T}\right]
$$

is the vector of constant estimated parameters of $\hat{N}$,

$$
\phi^{T}(t)=\left[-\hat{u}_{1}(t), \ldots-\hat{u}_{1}\left(t-n_{S}+1\right), \hat{y}_{1}(t+1), \ldots \hat{y}_{1}\left(t-n_{R}+1\right)\right]=\left[\phi_{\hat{u}_{1}}^{T}(t), \phi_{\hat{y}_{1}}^{T}(t)\right]
$$

and $\hat{y}_{1}(t+1)$ is given by eq. (10).

Of course this expression can be particularized for the case without internal positive coupling $\left(B_{M}=0\right)$ and for the case of the absence of feedback $(K=0)$.

Filtering the vector $\phi(t)$ through an asymptotically stable filter $L\left(q^{-1}\right)=\frac{B_{L}}{A_{L}}$, equation (24) for $\hat{\theta}=$ constant becomes:

$$
\begin{gathered}
\epsilon(t+1)=\frac{A_{M} A_{G} A_{K} G}{P_{f b-f f} L}[\theta-\hat{\theta}]^{T} \phi_{f}(t) \\
\phi_{f}(t)=L\left(q^{-1}\right) \phi(t) .
\end{gathered}
$$


Equation (28) has been used to develop the adaptation algorithms neglecting the non-commutativity of the operators when $\hat{\theta}$ is time-varying (however an exact algorithm can be derived in such cases see [10]).

Replacing the fixed estimated parameters by the current estimated parameters, equation (28) becomes the equation of the a-posteriori residual (adaptation) error $\varepsilon(t+1)$ (which is computed):

$$
\varepsilon(t+1 / \hat{\theta}(t+1))=\frac{A_{M} A_{G} A_{K}}{P_{f b-f f} L} G[\theta-\hat{\theta}(t+1)]^{T} \phi_{f}(t) .
$$

Equation (30) has the standard form for an a-posteriori adaptation error ([10]), which immediately suggests to use the same parametric adaptation algorithm given in equations (38) through (41). The stability of the algorithm has been analyzed in [37]

\subsection{Analysis of the Algorithms}

The equation for the a-posteriori adaptation error has the form

$$
\varepsilon(t+1)=H\left(q^{-1}\right)[\theta-\hat{\theta}(t+1)]^{T} \psi(t)
$$

where:

$$
H\left(q^{-1}\right)=\frac{A_{M} A_{G} A_{K}}{P_{f b-f f} L} G, \psi=\phi_{f} .
$$

Neglecting the non-commutativity of time-varying operators, one has the following result [36]:

Lemma 4.1: Assuming that eq. (31) represents the evolution of the a posteriori adaptation error and that the parameter adaptation algorithm (38) through (41) is used, one has:

$$
\begin{aligned}
\lim _{t \rightarrow \infty} \epsilon(t+1) & =0 \\
\lim _{t \rightarrow \infty} \frac{\left[\epsilon^{0}(t+1)^{2}\right]}{1+\psi(t)^{T} F(t) \psi(t)} & =0 \\
\|\psi(t)\| \text { is bounded } & \\
\lim _{t \rightarrow \infty} \varepsilon^{0}(t+1) & =0
\end{aligned}
$$

for any initial conditions $\hat{\theta}(0), \epsilon^{0}(0), F(0)$, provided that:

$$
H^{\prime}\left(z^{-1}\right)=H\left(z^{-1}\right)-\frac{\lambda_{2}}{2}, \max _{t}\left[\lambda_{2}(t)\right] \leq \lambda_{2}<2
$$

is a strictly positive real (SPR) transfer function.

Various choices can be considered for the filter $\mathrm{L}$ in order to satisfy the positive real condition. See $[38,37]$. It is important to remark that the positive real condition is strongly influenced by the presence of the feedback controller and its design. The best performances are in general obtained by taking $\mathrm{L}$ as an estimation of $H$ (see eq.(32)). Relaxation of the positive real condition by averaging arguments is discussed in [38] and by adding proportional adaptation in [39]. Filtering of the residual error can also be considered for satisfying the positive real condition, but this will modify the criterion which is minimized ([40,39]). Analysis of the algorithms when hypotheses $\mathrm{H} 2$ and $\mathrm{H} 3$ are violated can be found in [38].

\subsection{Use of the Youla- Kučera parametrization for adaptive feedforward disturbance compensation}

Since most of the adaptive feedforward vibration (or noise) compensation systems feature an internal "positive feedback" coupling between the compensator system and the correlated disturbance measurement which serves as reference, one may think building a stabilizing controller for this internal loop to which an additional filter will be added with the objective to enhance the disturbance attenuation capabilities while preserving the stabilization properties of the controller. In order to achieve this, instead of a standard IIR feedforward compensator on can use an Youla 


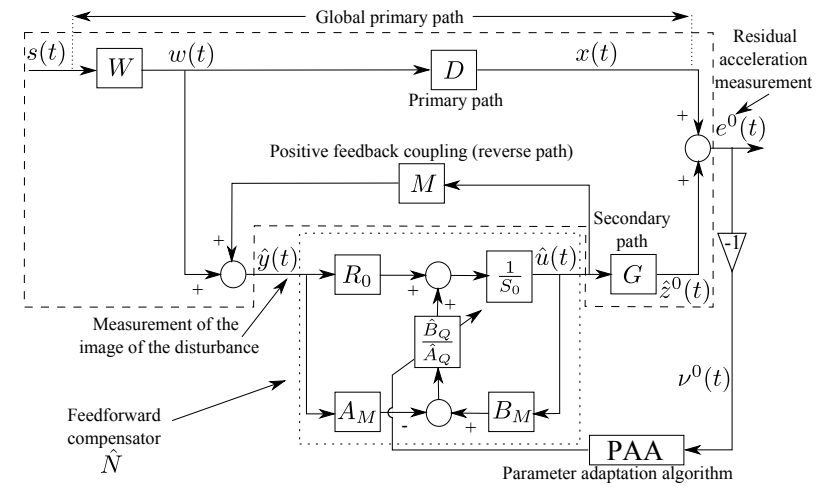

Figure 12. Adaptive feedforward disturbance compensation using Youla-Kučera parametrization

Kučera parametrization of the adaptive feedforward compensator. The central compensator will assure the stability of the internal positive feedback loop and its performance are enhanced in realtime by the direct adaptation of the parameters of the Youla-Kučera Q-filter. A block diagram of such adaptive feedforward compensator is shown in Fig. 12. FIR and IIR Q-filters can be used. Details of the specific algorithms can be found in [41, 42]. Comparisons between IIR, FIR YK, and IIR YK adaptive feedforward have been done. The conclusions of these comparison can be briefly summarized as follows:

- For the same level of performance IIR YK requires the lower number of adjustable parameters;

- IIR YK and FIR YK allow easily the incorporation of an initial stabilizing controller of any dimension while for IIR feedforward compensator this is more difficult;

- The performance of FIR YK is influenced by the performance of the initial model based stabilizing controller.

\section{SOME EXPERIMENTAL RESULTS - ATTENUATION OF BROAD BAND DISTURBANCES}

\subsection{Adaptive feedforward + fixed feedback attenuation of broad band disturbances}

A summary of various results obtained on the system described in Section II will be presented next. The adaptive feedforward filter structure for all the experiments has been $n_{R}=9, n_{S}=10$ (total of 20 parameters) and this complexity does not allow to verify the "perfect matching condition" (which requires more than 40 parameters). A feedback RS controller has been also introduced to test the potential improvement in performance.

Table II summarizes the global attenuation results for various configurations. Clearly, hybrid adaptive feedforward- fixed feedback scheme brings a significant improvement in performance with respect to adaptive feedforward compensation alone. This can be also seen on the power spectral densities shown in Figure $13^{12}$. A pseudo-random binary sequence (PRBS) excitation on the global primary path has been considered as the disturbance.

\begin{tabular}{lccccc}
\hline & $\begin{array}{c}\text { Feedback } \\
\text { only }\end{array}$ & $\begin{array}{c}\text { Feedforward } \\
\text { only } \\
\left(H_{\infty}\right)\end{array}$ & $\begin{array}{c}\text { Adaptive } \\
\text { Feedforward } \\
\text { only }\end{array}$ & $\begin{array}{c}\text { Feedback \& } \\
\text { Adaptive } \\
\text { Feedforward }\end{array}$ & $\begin{array}{c}\text { Feedforward } \\
\left(H_{\infty}\right) \\
\text { \& Feedback }\end{array}$ \\
\hline Attenuation [dB] & -14.40 & -14.70 & -16.23 & -20.53 & -18.42 \\
\hline
\end{tabular}

Table II. Global attenuation for various configurations

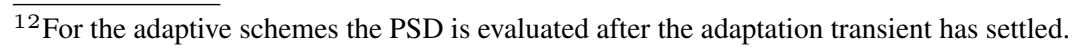




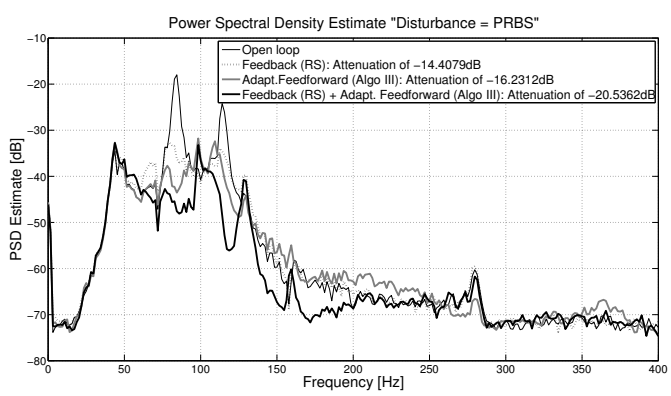

Figure 13. Power spectral densities of the residual acceleration (Disturbance $=$ PRBS).

It is important to point out that the design of a linear feedforward+feedback requires not only the perfect knowledge of the disturbance characteristics but also of the model of the primary path, while an adaptive approach does not require these informations. To illustrate the adaptation capabilities of the algorithms presented, a sinusoidal disturbance of $150 \mathrm{~Hz}$ has been added to the PRBS disturbance. Figure 14 shows the power spectral densities in open loop, using an adaptive algorithm and when the $H_{\infty}$ feedforward compensator which is not designed for this additional disturbance is used. One can remark that the hybrid adaptive feedforward-feedback scheme introduces a strong attenuation of the sinusoidal disturbance (larger than $30 \mathrm{~dB}$ ) without affecting other frequencies (compare with Figure 13) while the model based $H_{\infty}$ feedforward compensator + feedback controller has not been able to attenuate the sinusoidal disturbance.

Experimental time domain results obtained in open loop and with the hybrid control on the AVC system described in Section 2 are shown in Fig. 15 [36].

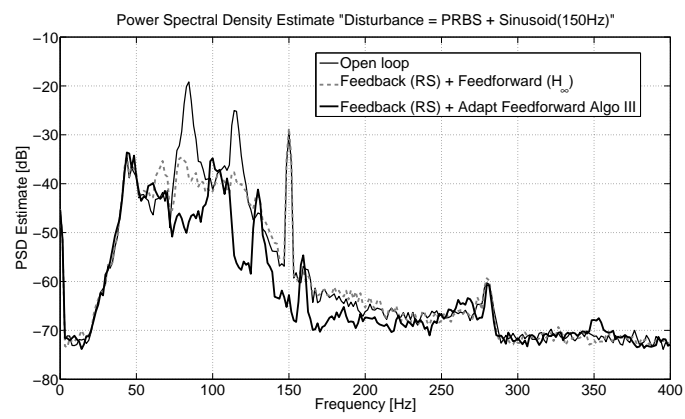

Figure 14. Power spectral densities when an additional sinusoidal disturbance is added (Disturbance $=$ PRBS + sinusoid)

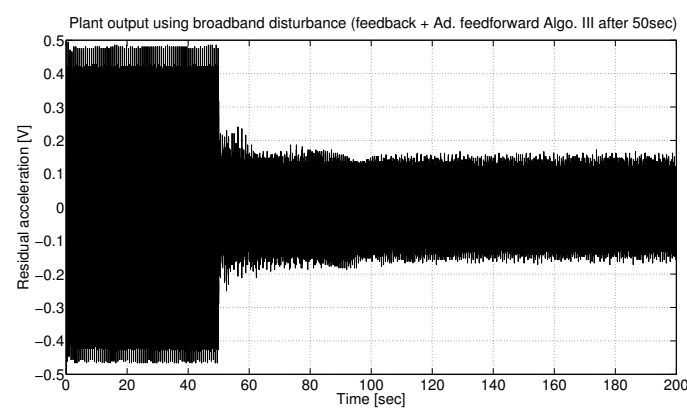

Figure 15. Real time results obtained with feedback controller and adaptive feedforward Algorithm III. 
The experiments have been carried on by first applying the disturbance in open loop during $50 \mathrm{~s}$ and after that closing the loop with the hybrid adaptive feedforward-feedback algorithms.

\section{CONCLUSION}

Adaptive attenuation of unknown disturbances appears as a well defined field and the results already available have a clear application impact. The paper has emphasized the advantage of using: the Youla-Kucera parametrization in the context of feedback and feedforward disturbance compensation, the stability based re-design of the filters used on the regressor vector and the use of matrix adaptation gains.

Important problems are nevertheless open and will require important research effort. We will mention just two:

- Adaptive feedback approach in the context of errors in the model of the secondary path [43].

- Combined adaptive feedforward and adaptive feedback.

\section{REFERENCES}

1. Alma M, Martinez JJ, Landau ID, Buche G. Design and tuning of reduced order h-infinity feedforward compensators for active vibration control. Control Systems Technology, IEEE Transactions on 2011; PP(99):18. DOI: 10.1109/TCST.2011.2119485.

2. Rotunno M, de Callafon R. Design of model-based feedforward compensators for vibration compensation in a flexible structure. Internal report, Dept. of Mechanical and Aerospace Engineering. University of California, San Diego 2003.

3. Elliott S. Signal Processing for Active Control. Academic Press: San Diego, California, 2001

4. Widrow B, Glover J JR, McCool J, Kaunitz J, Williams C, Hearn R, Zeidler J, Eugene Dong J, Goodlin R. Adaptive noise cancelling: Principles and applications. Proceedings of the IEEE dec 1975; 63(12):1692 - 1716, doi:10.1109/PROC.1975.10036.

5. Elliott S, Sutton T. Performance of feedforward and feedback systems for active control. Speech and Audio Processing, IEEE Transactions on may 1996; 4(3):214 -223, doi:10.1109/89.496217.

6. Jacobson C, Johnson J CR, McCormick D, Sethares W. Stability of active noise control algorithms. Signal Processing Letters, IEEE mar 2001; 8(3):74 -76, doi:10.1109/97.905944.

7. Zeng J, de Callafon R. Recursive filter estimation for feedforward noise cancellation with acoustic coupling. Journal of Sound and Vibration 2006; 291(3-5):1061 - 1079, doi:10.1016/j.jsv.2005.07.016.

8. Kuo S, Morgan D. Active noise control: a tutorial review. Proceedings of the IEEE jun 1999; 87(6):943 - 973, doi: $10.1109 / 5.763310$.

9. Hu J, Linn J. Feedforward active noise controller design in ducts without independent noise source measurements. IEEE transactions on control system technology 2000; 8(3):443-455.

10. Landau ID, Lozano R, M'Saad M, Karimi A. Adaptive control. 2nd edn., Springer: London, 2011.

11. Landau I, Zito G. Digital Control Systems - Design, Identification and Implementation. Springer: London, 2005.

12. Landau ID, Alma M, Constantinescu A, Martinez JJ, Noë M. Adaptive regulation-rejection of unknown multiple narrow band disturbances (a review on algorithms and applications). Control Engineering Practice 2011; 19(10): 1168 - 1181, doi:10.1016/j.conengprac.2011.06.005.

13. Tay TT, Mareels IMY, Moore JB. High Performance Control. Birkh auser Boston, 1997.

14. Bodson M, Douglas S. Adaptive algorithms for the rejection of sinusosidal disturbances with unknown frequency. Automatica 1997; 33:2213-2221.

15. Ben Amara F, Kabamba P, Ulsoy A. Adaptive sinusoidal disturbance rejection in linear discrete-time systems - Part I: Theory. Journal of Dynamic Systems Measurement and Control 1999; 121:648-654.

16. Valentinotti S. Adaptive rejection of unstable disturbances: Application to a fed-batch fermentation. Thèse de doctorat, École Polytechnique Fédérale de Lausanne April 2001.

17. Marino R, Santosuosso G, Tomei P. Robust adaptive compensation of biased sinusoidal disturbances with unknown frequency. Automatica 2003; 39:1755-1761.

18. Ding Z. Global stabilization and disturbance suppression of a class of nonlinear systems with uncertain internal model. Automatica 2003; 39(3):471 - 479, doi:10.1016/S0005-1098(02)00251-0.

19. Landau I, Constantinescu A, Rey D. Adaptive narrow band disturbance rejection applied to an active suspension an internal model principle approach. Automatica 2005; 41(4):563-574.

20. Kinney C, HFang, de Callafon R, Alma M. Robust estimation and automatic controller tuning in vibration control of time varying harmonic disturbances. Prepr. 18th IFAC World Congress, Milano, Italy 2011; :5401-5406.

21. Aranovskiy S, Freidovich LB. Adaptive compensation of disturbance formed as sums of sinusoidal signals with application to an active vibration control testbench. European Journal of Control 2013; .

22. Chen X, Tomizuka M. A minimum parameter adaptive approach for rejecting multiple narrow-band disturbances with application to hard disk drives. IEEE Transactions on Control System Technology Mar 2012; 20(2):408-415. 
23. Emedi Z, Karimi A. Fixed-order lpv controller design for rejection of a sinusoidal disturbance with time-varying frequency. 2012 IEEE Multi-Conference on Systems and Control, Dubrovnik, 2012; .

24. Marino R, Santosuosso G, Tomei P. Output feedback stabilization of linear systems with unknown additive output sinusoidal disturbances. European Journal of Control 2008; 14(2).

25. Landau I, Castellanos Silva A, Airimitoaie TB, Buche G, Noe M. Benchmark on adaptive regulation - rejection of multiple unknown/time-varying multiple narrow band disturbances. European Journal of Control 2013;

26. Marcos T. The straight attraction. Motion control 2000; 13:29-33.

27. Anderson B. From Youla-Kucera to identification, adaptive and nonlinear control. Automatica 1998; 34:1485-1506.

28. Tsypkin Y. Stochastic discrete systems with internal models. Journal of Automation and Information Sciences 1997; 29(4\&5):156-161.

29. Landau I, Alma M, Martinez J, Buche G. Adaptive suppression of multiple time-varying unknown vibrations using an inertial actuator. Control Systems Technology, IEEE Transactions on nov 2011; 19(6):1327 -1338, doi: 10.1109/TCST.2010.2091641.

30. Procházka H, Landau ID. Pole placement with sensitivity function shaping using 2nd order digital notch filters. Automatica 2003; 39(6):1103 - 1107, doi:10.1016/S0005-1098(03)00067-0.

31. Nehorai A. A minimal parameter adaptive notch filter with constrained poles and zeros. IEEE Trans. Acoust., Speech, Signal Processing 1985; ASSP-33:983-996.

32. M'Sirdi N, Tjokronegoro H, Landau I. An rml algorithm for retrieval of sinusoids with cascaded notch filters. Acoustics, Speech, and Signal Processing, 1988. ICASSP-88., 1988 International Conference on, 1988; 2484 2487 vol.4, doi:10.1109/ICASSP.1988.197147.

33. Airimitoaie TB, Castellanos Silva A, Landau ID. Indirect adaptive regulation strategy for the rejection of time varying narrow-band disturbances applied to a benchmark problem. European Journal of Control 2013; .

34. Airimitoaie TB, Landau ID. Indirect adaptive attenuation of multiple narrow-band disturbances applied to active vibration control. IEEE Transactions on Control System Technology 2013; .

35. Castellanos Silva A, Landau ID, Airimitoaie TB. Direct adaptive rejection of unknown time-varying narrow band disturbances applied to a benchmark problem. European Journal of Control 2013; .

36. Alma M, Landau I, Martinez J, Airimiţoaie T. Hybrid adaptive feedforward-feedback compensation algorithms for active vibration control systems. 50th IEEE Conference on Decision and Control and European Control Conference 2011.

37. Alma M, Landau ID, Airimitoaie TB. Adaptive feedforward compensation algorithms for avc systems in the presence of a feedback controller. Automatica 2012; 48(10):982 - 985.

38. Landau I, Alma M, Airimiţoaie T. Adaptive feedforward compensation algorithms for active vibration control with mechanical coupling. Automatica 2011; 47(10):2185 - 2196, doi:10.1016/j.automatica.2011.08.015.

39. Airimitgoaie TB, Landau ID. Improving adaptive feedforward vibration compensation by using integral+proportional adaptation. Automatica 2013; 49(5):1501-1505, doi:10.1016/j.automatica.2013.01.025.

40. Montazeri AD, Postham J. A new adaptive recursive rls-based fast-array iir filter for active noise and vibration control systems. Signal Processing 2011; $91: 98$ - 113.

41. Landau I, Airimiţoaie T, Alma M. A youla-kučera parametrized adaptive feedforward compensator for active vibration control with mechanical coupling. Automatica 2012. In review.

42. Landau ID, Airimitoaie TB, Alma M. IIR Youla-Kucera parameterized adaptive feedforward compensators for active vibration control with mechanical coupling. IEEE Transactions on Control System Technology 2013; .

43. Mullhaupt P, Bonvin D. Asymptotic rejection of nonvanishing disturbances despite plant-model mismatch. Int. J. of Adapt. Control Signal Process 2012; 26(12):1090-1110. 